\title{
Dedicated bifurcation analysis: basic principles
}

\author{
Joan C. Tuinenburg • Gerhard Koning • \\ Andrei Rareş • Johannes P. Janssen • \\ Alexandra J. Lansky $\cdot$ Johan H. C. Reiber
}

Received: 24 December 2010/Accepted: 30 December 2010/Published online: 17 February 2011

(C) The Author(s) 2011. This article is published with open access at Springerlink.com

\begin{abstract}
Over the last several years significant interest has arisen in bifurcation stenting, in particular stimulated by the European Bifurcation Club. Traditional straight vessel analysis by QCA does not satisfy the requirements for such complex morphologies anymore. To come up with practical solutions, we have developed two models, a Y-shape and a T-shape model, suitable for bifurcation QCA analysis depending on the specific anatomy of the coronary bifurcation. The principles of these models are described in this paper, as well as the results of validation studies carried out on clinical materials. It can be concluded that the accuracy, precision and applicability of these new bifurcation analyses are conform the general guidelines that have been set many years ago for conventional QCA-analyses.
\end{abstract}

Keywords Quantitative coronary arteriography · Bifurcation analysis

J. C. Tuinenburg ( $₫)$ · G. Koning · A. Rareş .

J. P. Janssen - J. H. C. Reiber

Division of Image Processing (LKEB),

Department of Radiology, Leiden University Medical

Center, Leiden, The Netherlands

e-mail: J.C.Tuinenburg@lumc.nl

\section{A. J. Lansky}

Yale School of Medicine, Yale University,

New Haven, CT, USA

\section{Introduction}

In interventional cardiology, Quantitative Coronary Arteriography (QCA) has been used for on-line vessel sizing for the selection of the interventional devices and the assessment of the efficacy of the individual procedures, for the on-line selection of patients to be included or excluded in clinical trials based on quantitative parameters (e.g. small vessel disease), and for training purposes. But, in particular, QCA has been applied worldwide in core laboratories and clinical research sites to study the efficacy of the procedures and devices in smaller and larger patient populations in off-line situations. Newer developments are directed towards the 3D reconstruction of the coronary arteries and the fusion with IVUS or OCT [1-3].

The goal of this paper is to provide a brief overview of the basic principles of a modern QCA software package, particularly on the field of coronary bifurcation analysis. This is best illustrated by the QAngio ${ }^{\circledR}$ XA package (Medis medical imaging systems bv, Leiden, the Netherlands).

\section{Bifurcation analysis}

With the expanding practice of stenting coronary bifurcation lesions worldwide [4], the need for reliable, standardized and reproducible quantitative bifurcation analyses became apparent. Hence, the 
QAngio XA version7.2 bifurcation application was developed, which contains two bifurcation models: a T-shape bifurcation model (suitable for bifurcations with a standard side branch structure: Fig. 1a) and a Y-shape bifurcation model (suitable for bifurcations with distal branches of equal size: Fig. 1b). The particular advantage of these models is that they combine the proximal and two distal vessel segments with the bifurcation core, resulting in a total of two or three sections (depending on the model type), all derived from one analysis procedure, such that each of these sections has its own diameter function and associated parameter data.

To be able to start a bifurcation analysis, an analysis frame is chosen from the selected image run in which the target vessel is fully contrast-filled
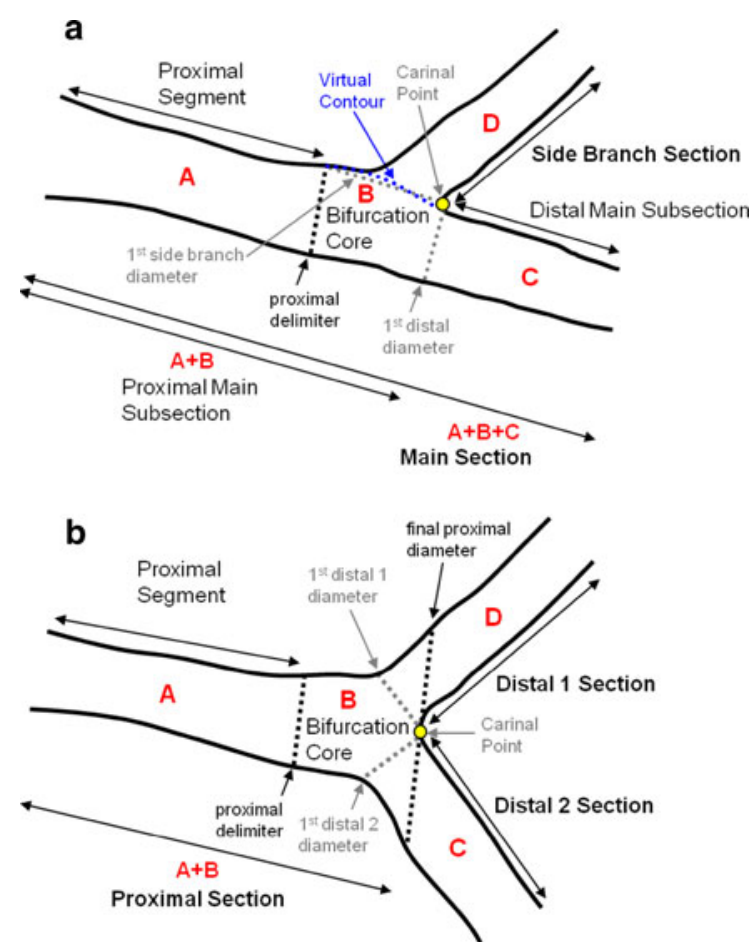

Fig. 1 Schemes of the a T-shape model and $\mathbf{b} Y$-shape model, explaining the segments, proximal delimiter, interpolated contour and sections terminology. For each model, four segments that represent the building blocks of the models are generated by the software. a Using the T-shape model, the arterial and reference diameters of the ostium of the side branch and the whole main section (including the transition within the bifurcation core) can be accurately determined. b Using the $Y$-shape model, the arterial and reference diameters up to the carinal point and in the distal 1 and 2 sections can be determined accurately (usually second or third cardiac cycle following contrast injection) and in a "stable" position (preferably end-diastole), i.e. minimal motion with respect to neighboring frames to prevent motion blur [5, 6]. As a first step, the user selects three pathline points in the image to define the arterial bifurcation segment: a start point in the proximal segment and one end point in each of the two distal segments are required. Subsequently, independent of the model type, two pathlines through the bifurcation segment of interest are computed automatically (Fig. 2a) based on the wavefront propagation principle ('the wavepath approach') [7, 8], followed by the automated detection of the arterial contours of all three vessel segments at once (Fig. 2b).

This arterial contour-detection procedure is carried out in two iterations relative to a model (here explained for a straight vessel, to keep things simple). In the first iteration, the pathline is used as the model (Fig. 3a). To detect the contours, scanlines are defined perpendicular to the model (Fig. 3b). For each point or pixel along such a scanline, the corresponding edge-strength value (local change in brightness level) is computed as the weighted sum of the corresponding values of the first- and second-derivative functions applied to the brightness values along these scanlines (Fig. 4). The resulting edge-strength values are input to the socalled minimum cost contour-detection algorithm (MCA) [9-11], which searches for an optimal contour path along the entire segment (Fig. 3c). The individual left and right vessel contours, detected in the first iteration, now serve as models for the MCA procedure in the second iteration, resulting in the initially detected arterial contours (Fig. 3d). If the operator does not agree with one or more parts of the initially detected contours, these can be edited/corrected in various ways. In a similar manner, the arterial contours are detected for the bifurcation analysis, resulting in three contours: the left, right and middle contour (Fig. 2b).

The bifurcation core of the T-shape model is defined as the area between the automatically determined proximal delimiter in the proximal main subsection (of which the position is independent of the presence of a lesion) and the carinal point, which is flanked at one side by the first diameter of the distal main subsection (identical to the distal main segment) and at the other side by the interpolated (virtual) 
Fig. 2 An example of the $T$-shape model bifurcation analysis. a The three pathline points with the two detected bifurcation pathlines. b The three detected arterial contours. c The final analysis contours, plaque filling and the two corresponding diameter functions of the main and side branch sections a

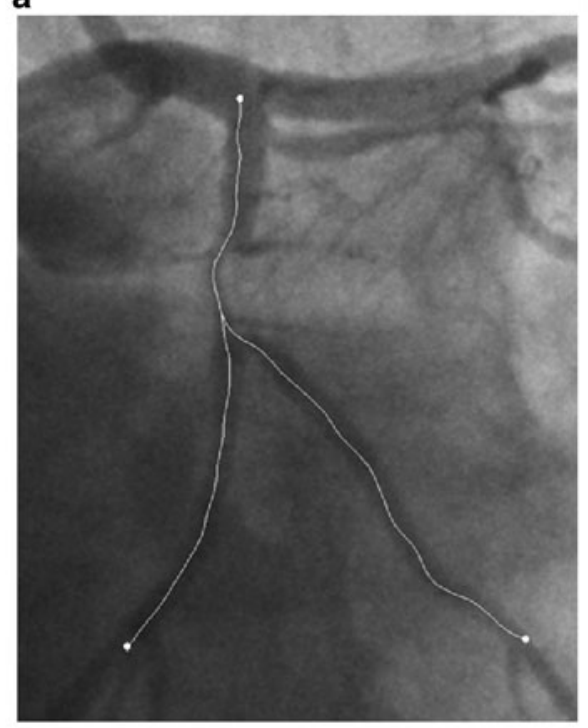

b

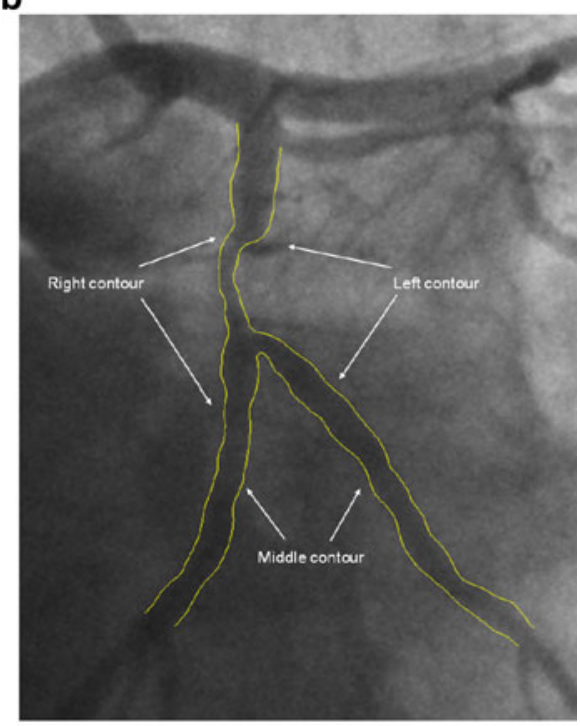

c

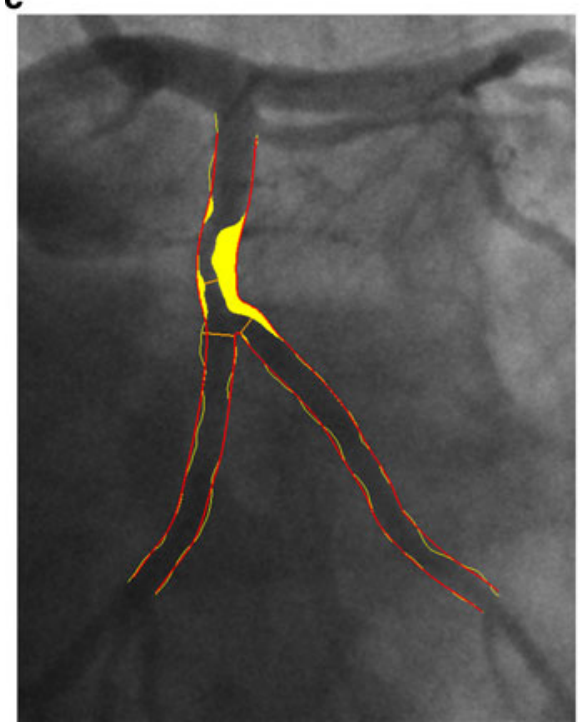

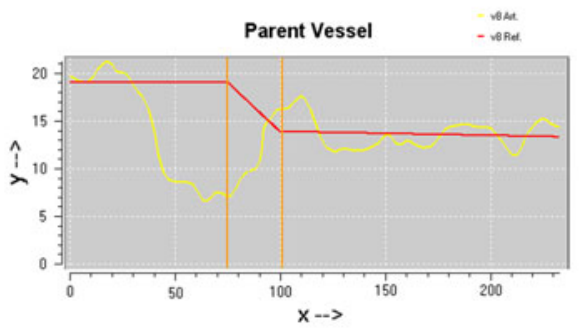

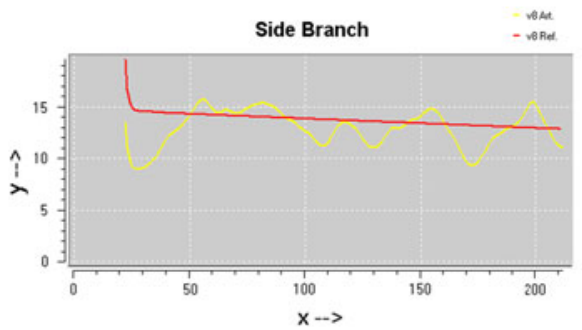

contour between the proximal and distal main segments (Fig. 1a). From the arterial contours and the interpolated contour, two sections are defined: the main section (i.e. the proximal main, distal main and bifurcation core segments merged) and the side branch section (identical to the side branch segment) (Fig. 1a). From the left- and right-hand contours of the main section, an arterial diameter function is calculated following the conventional straight analysis approach (Fig. 5a, yellow graph), while for the side branch section the ostial analysis approach is followed [12], making sure that the arterial diameters at the ostium of the side branch are measured properly (Fig. 5b, yellow graph).

The most widely used parameter to describe the severity of an obstruction is the percentage of diameter stenosis. Calculation of this parameter requires that a reference diameter value is computed, for which two options are available: (1) a userdefined reference diameter as positioned by the user at a so-called "normal" portion of the vessel, and (2) the automated or interpolated reference diameter value. In practice, this last approach is preferred because it requires no user interaction and takes care 


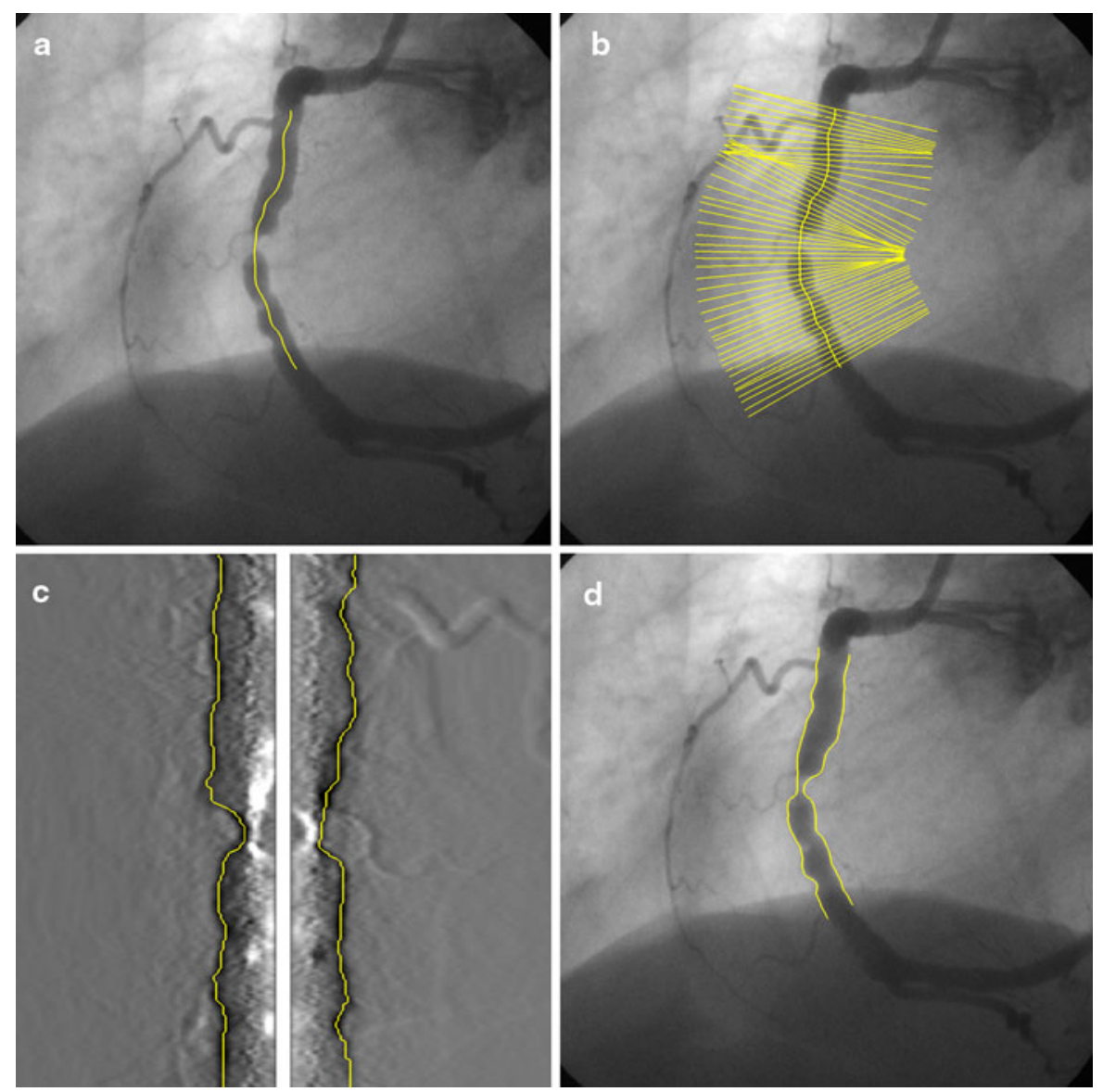

Fig. 3 Basic principles of the minimum cost contour-detection algorithm (MCA). a Initial segment with pathline, b scanlines defined, c straightened for analysis; contours calculated, $\mathbf{d}$ contours returned to initial image

of any tapering of the vessel. For a conventional straight analysis, a reference diameter function is calculated by an iterative regression technique (excluding the influence of any obstructive or ectatic area) and displayed in the diameter function as a (slightly tapering) horizontal straight line (Fig. 5a, red line), which represents the best approximation of the vessel size before the occurrence of a focal narrowing. Now that the reference diameter function is known, reference contours can be reconstructed around the actual vessel segment, representing the original size and shape of the vessel before any disease occurred. The value of the reference diameter function at the location of the obstruction diameter equals the reference diameter, so that neither overestimation nor underestimation occurs. Finally, from the reference diameter and the obstruction diameter, the percent diameter stenosis is calculated.
However, due to the "step down" phenomenon caused by the bifurcating vessels, it is not a trivial task to derive a suitable reference diameter function for the entire main section [13]. Therefore, the calculation of the reference diameter function is based on each of the three segments separately. By this approach, it is assured that both the proximal and distal main (interpolated) reference diameter functions are only based on the arterial diameters outside the bifurcation core. Finally, the reference diameter function of the bifurcation core is based on the reconstruction of a smooth transition between the proximal and distal vessel diameters. As a result, the reference diameter function graph of the entire main section will be displayed as one function, which is composed of three different straight reference lines that are linked together (Fig. 2c, right above). For the side branch section an "ostial" reference diameter 


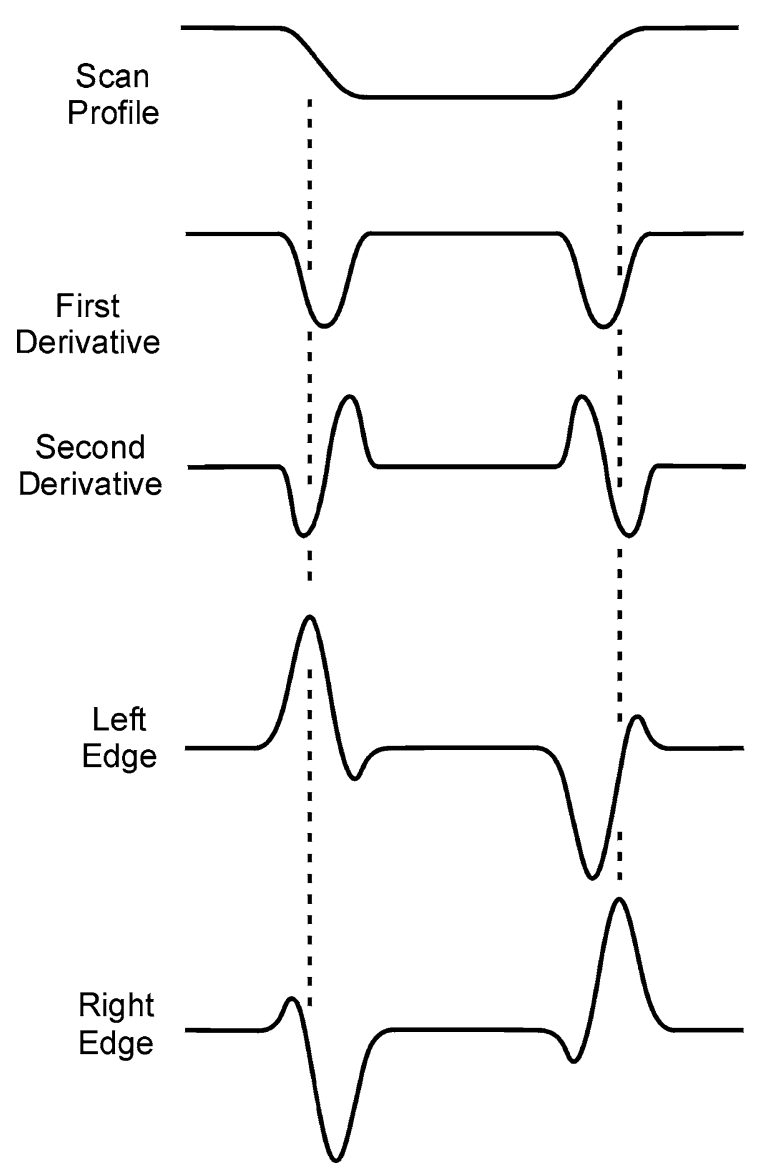

Fig. 4 The edge-strength values along a scanline. Schematic presentation of the brightness profile of an arterial vessel assessed along a scanline perpendicular to the local pathline direction and the computed 1st-derivative, 2nd-derivative, and the combinations of these 1st- and 2nd-derivative function; the maximal values of the last functions determine the edge positions function calculation is used (Fig. 5b, red line) [12], which is displayed as one function, which is straight and slightly curved proximally (Fig. 2c, right under).

The bifurcation core of the Y-shape model is defined as the area between the automatically determined proximal delimiter in the proximal section and the carinal point (Fig. 1b). From the arterial contours and by using the carinal point, three sections are defined: the proximal section (i.e. the proximal and bifurcation core segments merged), the distal 1 section and the distal 2 section (Fig. 1b). For each of these sections the corresponding arterial diameter functions are calculated following the conventional straight analysis approach [12]. This method guarantees that within the bifurcation core the arterial diameters are measured in their fullest extent (e.g. important for skirt stenting).

In order to derive a suitable reference diameter function for each section, again the calculation of the reference diameter function is based on each of the segments separately. The reference diameter function of the bifurcation core itself is based on reconstructed reference contours between the proximal segment and two distal sections. As a result, the reference diameter function graph of the entire proximal section will be displayed as one function, which is straight for the proximal segment and curved in the bifurcation core. The two reference diameter functions of the distal sections will each be displayed as one function and are straight (Fig. 6).

The reported bifurcation analysis results of all sections (both models) will be a complete listing of the angiographic parameters similar to the conventional
Fig. 5 Examples of the a straight analysis and b ostial analysis, each with their own contours and specific diameter function (graph)
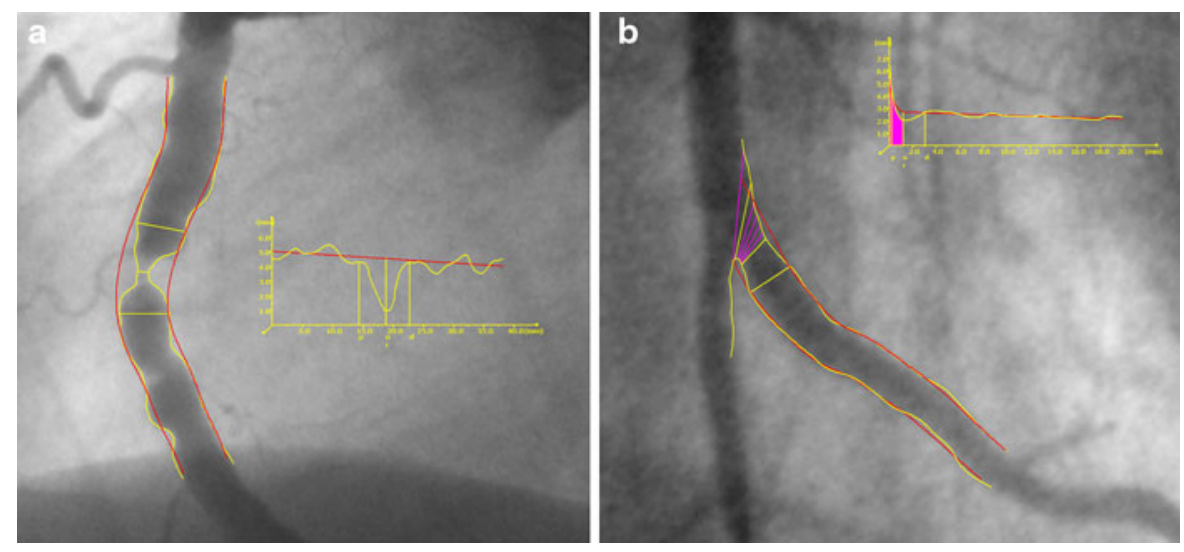
Fig. 6 An example of the $Y$-shape model bifurcation analysis. The final analysis contours, plaque filling and the three corresponding diameter functions of the proximal, distal 1 and distal 2 sections, respectively a

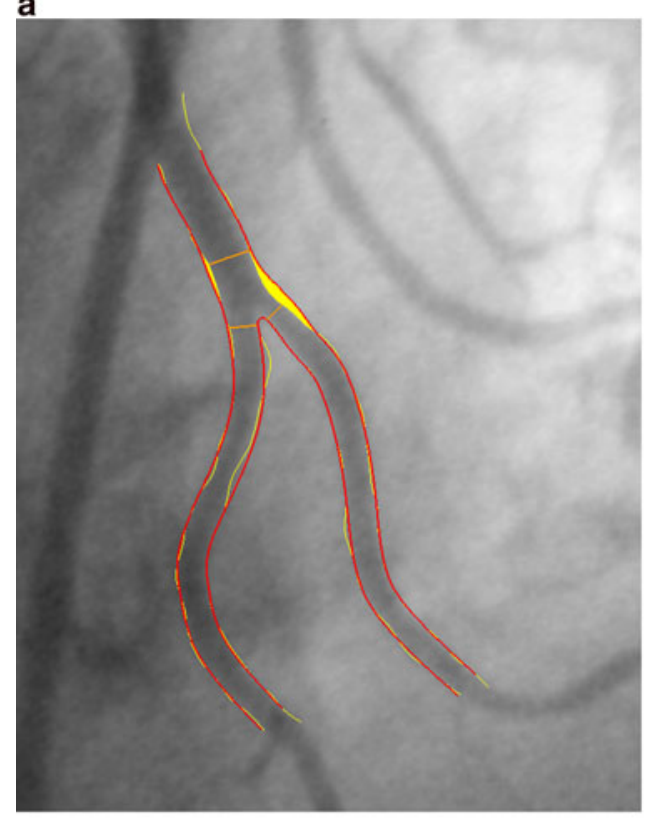

b
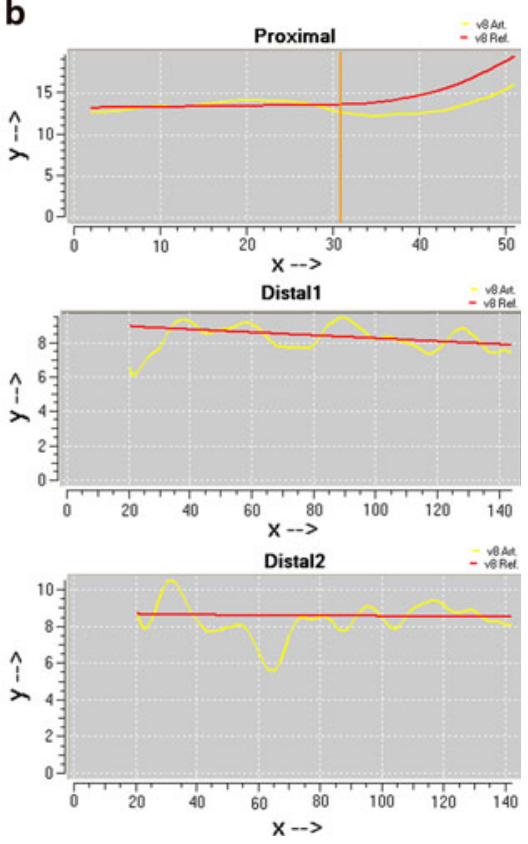

Fig. 7 A schematic overview of the edge segment analysis for the bifurcation's T-shape analysis model

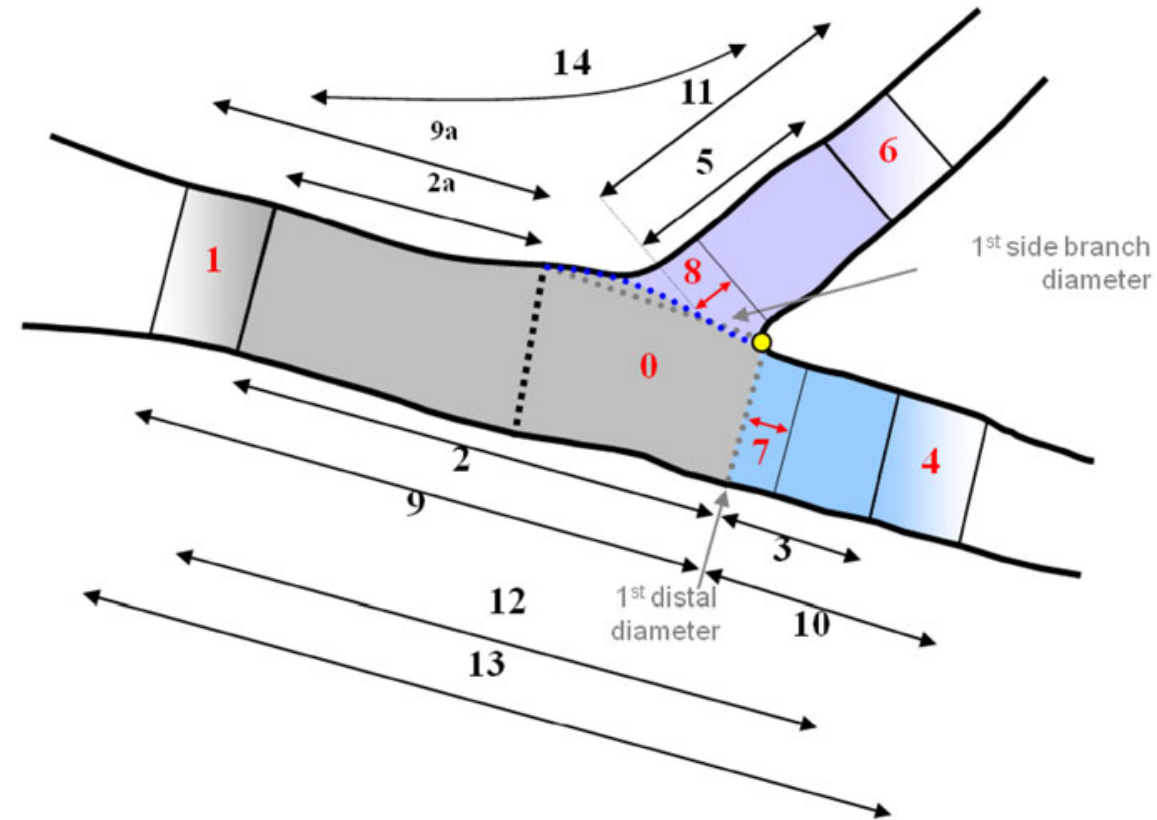

0 - Bif Core

1 - Edge Proximal

2 - Stented Proximal Main

$2 a-$ Stented Proximal Segment

3 - Stented Distal Main

4 - Edge Distal Main

5 - Stented Side Branch

6 - Edge Side Branch
7 - Ostium Distal Main ( 3 or $5 \mathrm{~mm}$ )

8 - Ostium Side Branch ( 3 or $5 \mathrm{~mm}$ )

9 - Analyzed Proximal Main

9a-Analyzed Proximal Segment

10-Analyzed Distal Main

11- Analyzed Side Branch

12-Stented Main

13-Analyzed Main

14 - Stented Proximal-Side Branch 
Table 1 Guidelines for systematic and random errors of a state-of-the-art QCA system

\begin{tabular}{lllr}
\hline Type of study & Systemic error $(\mathrm{mm})$ & \multicolumn{2}{l}{ Random error $(\mathrm{mm})$} \\
\cline { 3 - 4 } & & Guidelines & Result ranges \\
\hline Plexiglass phantom, off patient & $<0.10$ & $0.10-0.13$ & $0.06-0.12$ \\
Plexiglass phantom, on patient & $<0.10$ & $0.10-0.13$ & $0.10-0.11$ \\
\hline & & Guidelines & $\mathrm{D}_{\text {obs }}$ \\
\hline Intra-observer variabilities & $0.10-0.15$ & 0.10 & 0.11 \\
Inter-observer variabilities & $0.10-0.15$ & 0.13 \\
Short-term variabilities & $0.15-0.25$ & 0.13 \\
Medium-term variabilities & $0.20-0.30$ & 0.22 \\
Inter-core lab variability & & 0.18 & 0.14 \\
\hline
\end{tabular}

Table 2 The intra-observer differences (Mean \pm StDev) of the obstruction and reference diameters, for both the T-and Y-shape models

\begin{tabular}{lllrr}
\hline & & Prox main (incl bif core) & Dist main & Side branch \\
\hline T-shaped model $(\mathrm{n}=18)$ & Dobs $(\mathrm{mm})$ & $0.01 \pm 0.03$ & $-0.01 \pm 0.04$ & $0.01 \pm 0.05$ \\
& Dref $(\mathrm{mm})$ & $0.08 \pm 0.10$ & $0.01 \pm 0.08$ & $0.04 \pm 0.10$ \\
Y-shaped model $(\mathrm{n}=18)$ & Dobs $(\mathrm{mm})$ & $0.00 \pm 0.03$ & $0.02 \pm 0.08$ & $-0.01 \pm 0.06$ \\
& Dref $(\mathrm{mm})$ & $0.03 \pm 0.11$ & $-0.03 \pm 0.09$ & $0.02 \pm 0.10$ \\
\hline
\end{tabular}

All values are very low, demonstrating the high reproducibility of the analyses with both models, including lesions at the bifurcation core

straight QCA, including the obstruction, reference, minimum, maximum, and mean diameters and areas, the percent diameter and area stenosis as well as the vessel and lesion lengths.

Additionally, an option for edge segment analysis over the bifurcation (both models) will be available for the assessment of (drug eluting) stent segments and the corresponding stent- and ostial edge segments (Fig. 7; T-shape). For each of these (sub)segments the complete parameter set including some edge specific parameters (e.g. MLD position relative to the stent boundary or segment start position, etc.) will be reported, to allow to study the regression and progression of the bifurcation lesion to the fullest.

\section{Validation}

Whichever QCA analytical software package is being used, it will always produce numbers describing the morphology of the coronary segment analyzed. However, validation studies must demonstrate the true strengths and weaknesses, as well as the clinical validity of such analytical package.

For a QCA technique to be acceptable, guidelines for systematic and random error (a.k.a. accuracy and precision) values of absolute vessel dimensions have been established. These guidelines are shown in Table 1 [14]. Over the years we have carried out many validation studies, which all have been presented in the international literature [e.g.: 14-16]. Considering the bifurcation analysis, the most relevant bifurcation validation studies are of the bifurcation's diameter functions [17] and the intra-observer variability of the $\mathrm{T}$ - and Y-shape models (see Table 2).

\section{Conclusion}

Semi-automated segmentation techniques are able to detect the luminal boundaries of coronary arteries from two-dimensional digital X-ray arteriograms after minimal user interaction. QCA can be used in 
an off-line mode for clinical research studies and in an on-line mode during the interventional procedure to support the clinical decision-making process. New approaches have become available for more extensive analyses such as the coronary bifurcation analysis.

Open Access This article is distributed under the terms of the Creative Commons Attribution Noncommercial License which permits any noncommercial use, distribution, and reproduction in any medium, provided the original author(s) and source are credited.

Conflict of interest Joan C. Tuinenburg and Gerhard Koning are employed by Medis medical imaging systems bv and have a research appointment at the Leiden University Medical Center. Andrei Rareş and Johannes P. Janssen have a research appointment at the Leiden University Medical Center. Alexandra J. Lansky is Director of the Yale Cardiovascular Research Group and Director of the Yale Women's Heart Center of the Yale School of Medicine. Johan H.C. Reiber is the CEO of Medis medical imaging systems bv, and has a parttime appointment at the LUMC as Prof of Medical Imaging.

\section{References}

1. Tu S, Koning G, Jukema W, Reiber JHC (2010) Assessment of obstruction length an optimal viewing angle from biplane X-ray angiograms. Int $\mathrm{J}$ Cardiovasc Imaging 26:5-17

2. Tu S, Huang Z, Koning G, Cui K, Reiber JHC (2010) A novel three-dimensional quantitative coronary angiography system: in vivo comparison with intravascular ultrasound for assessing arterial segment length. Catheter Cardiovasc Interv 76:291-298

3. Tu S, Holm NR, Koning G, Huang Z, Reiber JHC (2011) Fusion of 3D QCA and IVUS/OCT. Int $\mathbf{J}$ Cardiovasc Imaging. doi:10.1007/s10554-011-9809-2

4. Lefevre T, Louvard Y, Morice MC, Loubeyre C, Piechaud JF, Dumas P (2001) Stenting of bifurcation lesions: a rational approach. J Interv Cardiol 14(6):573-585

5. Lespérance J, Bilodeau L, Reiber JHC, Koning G, Hudon G, Bourassa M (1998) Issues in the performance of quantitative coronary angiography in clinical research trials. In: Reiber JHC, van der Wall EE (eds) What's new in cardiovascular imaging?. Kluwer Academic Publishers, Dordrecht, pp 31-46

6. Beleslin B, Ostojic M, Nedeljkovic M et al (1995) Assessment of the reproducibility of the basic parameters obtained from quantitative coronary arteriography analyses. Eur Heart J 16(abstract Suppl):296
7. Janssen JP, Koning G, de Koning PJ, Tuinenburg JC, Reiber JHC (2002) A novel approach for the detection of pathlines in X-ray angiograms: the wavefront propagation algorithm. Int J Cardiovasc Imaging 18(5):317-324

8. Janssen JP, Koning G, de Koning PJ, Tuinenburg JC, Reiber JH (2004) Validation of a new method for the detection of pathlines in vascular $\mathrm{x}$-ray images. Invest Radiol 39(9):524-530

9. Reiber JHC, Serruys PW, Kooijman CJ, Wijns W, Slager CJ, Gerbrands JJ et al (1985) Assessment of short-, medium-, long-term variations in arterial dimensions from computer-assisted quantitation of coronary cineangiograms. Circulation 71(2):280-288

10. Reiber JHC, van der Zwet PM, Koning G, von Land CD, van Meurs B, Gerbrands JJ et al (1993) Accuracy, precision of quantitative digital coronary arteriography: observer-, short-, medium-term variabilities. Cathet Cardiovasc Diagn 28(3):187-198

11. Reiber JHC, Koning G, Dijkstra J, Wahle A, Goedhart B, Sheehan FH et al (2001) Angiography and intravascular ultrasound. In: Sonka M, Fitzpatrick JM (eds) Handbook of medical imaging_volume 2: medical image processing and analysis. SPIE Press, Belligham, pp 711-808

12. Reiber JHC, Tuinenburg JC, Koning G, Janssen JP, Rares A, Lansky AJ, Goedhart B (2009) Ch 2.2 Quantitative coronary arteriography. In: Oudkerk M, Reiser MF (eds) Medical radiology_Diagnostic imaging, volume Coronary radiology, 2nd revised edn. Springer-Verlag, Berlin/ Heidelberg, pp 41-65

13. Lansky AJ, Tuinenburg JC, Costa M, Maeng M, Koning G, Popma J et al (2009) Quantitative angiographic methods for bifurcation lesions: A consensus statement from the European Bifurcation Group. Catheter Cardiovasc Intervent 73:258-266

14. Reiber JHC, Koning G, von Land CD, van der Zwet PM (1994) Why and how should QCA systems be validated? In: Reiber JHC, Serruys PW (eds) Progress in quantitative coronary arteriography. Kluwer Academic Publishers, Dordrecht, pp 33-48

15. Reiber JHC, von Land CD, Koning G, van der Zwet PM, van Houdt R, Schalij M et al (1994) Comparison of accuracy and precision of quantitative coronary arterial analysis between cinefilm and digital systems. In: Reiber JHC, Serruys PW (eds) Progress in quantitative coronary arteriography. Kluwer Academic Publishers, Dordrecht, pp 67-85

16. Beauman G, Reiber JHC, Koning G, van Houdt R, Vogel R (1994) Angiographic core laboratories analyses of arterial phantom images: Comparative evaluations of accuracy and precision. In: Reiber JHC, Serruys PW (eds) Progress in quantitative coronary arteriography. Kluwer Academic Publishers, Dordrecht, pp 87-104

17. Janssen JP, Rares A, Tuinenburg JC, Koning G, Lansky AJ, Reiber JHC (2010) New approaches for the assessment of vessel sizes in quantitative (cardio-)vascular X-ray analysis. Int J Cardiovasc Imaging 26(3):259-271 